\title{
Spin susceptibility of spin-orbit-coupled Fermi superfluids
}

\author{
M. Iskin \\ Department of Physics, Koç University, Rumelifeneri Yolu, 34450 Sartyer, Istanbul, Turkey
}

(Received 9 February 2018; published 21 May 2018)

\begin{abstract}
Under the self-consistent mean-field approach for the BCS-BEC crossover problem, we derive a closed-form analytical expression for the general spin response of noncentrosymmetric Fermi superfluids with arbitrary spinorbit coupling and Zeeman fields. In addition to the paramagnetic, i.e., the Pauli intrahelicity and Van Vleck type interhelicity, contributions to the spin-susceptibility tensor that have normal-state counterparts, we identify a diamagnetic interhelicity contribution that is unique to the superfluid state. Our extensive numerical calculations for the Weyl, Rashba, and equal Rashba-Dresselhaus spin-orbit couplings illustrate that it is this diamagnetic contribution that grows gradually with pairing and cancels precisely the Van Vleck contribution away from the BCS regime in general.
\end{abstract}

DOI: 10.1103/PhysRevA.97.053613

\section{INTRODUCTION}

Having two-dimensional metals with a weak Rashba spinorbit coupling (SOC) in mind, the BCS theory of the superconducting state without inversion symmetry [1,2] was initially developed to answer how the lack of spatial reflection symmetry can affect properties of a superconductor. One of their main conclusions is that, by lifting the twofold spin degeneracy, and hence mixing the spin-singlet and spin-triplet components in the wave function of the Cooper pairs, SOC gives rise to a nonzero and rather high spin response for the ground state of noncentrosymmetric superconductors. Since the spin susceptibility is commonly used as a means to probe and distinguish the spin-singlet and spin-triplet pairings, following the original footsteps [2], the spin response of such superconductors and superfluids (SFs) has been the main subject of many papers, with broad interest first in the condensed-matter physics community [3-7] and then in the cold-atom one [8-10].

In this paper, we revisit the spin response of uniform noncentrosymmetric Fermi SFs with arbitrary SOC and Zeeman fields, and we derive a closed-form analytical expression for the generalized spin-susceptibility tensor $\chi_{i j}$ through the derivative expansion of the mean-field thermodynamic potential. Our calculation yields three distinct contributions denoted by $\chi_{i j}=$ $\chi_{i j}^{\text {intra }}+\chi_{i j(a)}^{\text {inter }}+\chi_{i j(b)}^{\text {inter }}$. In addition to the usual paramagnetic Pauli intrahelicity contribution $\chi_{i j}^{\text {intra }}$ and the paramagnetic Van Vleck-type interhelicity contribution $\chi_{i j(a)}^{\text {inter }}$ that have normal-state counterparts, we find a diamagnetic interhelicity contribution $\chi_{i j(b)}^{\text {inter }}$ that is unique to the SF state. By performing extensive numerical calculations for the Weyl, Rashba, and equal Rashba-Dresselhaus (ERD) SOCs, we illustrate that while the spin response is dominated quite strongly by $\chi_{i j(a)}^{\text {inter }}$ in the BCS limit, it is the diamagnetic contribution $\chi_{i j(b)}^{\mathrm{inter}}$ that grows gradually with pairing and cancels precisely the paramagnetic contribution $\chi_{i j(a)}^{\text {inter }}$ away from the BCS regime. Thus, the existence of a nonzero ground-state spin response is not truly a measure of the SOC-induced spin-triplet component of the SF order parameter. However, since the $\chi_{i j(b)}^{\text {inter }}$ contribution has a strong peak in the vicinity of unitarity, observation of its diamagnetic effect is within the current reach of recent cold-atom experiments [11-15], as it accounts for the physical mechanism that forms spinless molecules from Cooper pairs in the BEC limit.

The rest of the paper is organized as follows. Starting with an introductory discussion of the noninteracting problem in Sec. II, we first derive closed-form analytical expressions for uniform SFs in Sec. III, and then we present their numerical analysis in Sec. IV. The paper ends with a brief summary of our conclusions in Sec. V.

\section{NONINTERACTING FERMI GASES}

To gain initial insight into the SF Fermi gases, let us first discuss the spin-susceptibility tensor of noninteracting Fermi gases that are described by the generic Hamiltonian density $H_{\mathbf{k}}=\xi_{\mathbf{k}} \sigma_{0}-\mathbf{Z}_{\mathbf{k}} \cdot \sigma$ in momentum $(\mathbf{k})$ and $\operatorname{spin}(\sigma)$ space. Here, $\xi_{\mathbf{k}}=\epsilon_{\mathbf{k}}-\mu$ is the usual free-particle dispersion $\epsilon_{\mathbf{k}}=k^{2} /(2 m)$ (in units of $\hbar \rightarrow 1$ the Planck constant) shifted by the chemical potential $\mu$, and $\mathbf{Z}_{\mathbf{k}}=\mathbf{h}-\mathbf{d}_{\mathbf{k}}$ is effectively a k-dependent Zeeman field combining the true Zeeman field $\mathbf{h}=\sum_{i} h_{i} \widehat{\boldsymbol{i}}$ with the SOC field $\mathbf{d}_{\mathbf{k}}=\sum_{i} d_{\mathbf{k}}^{i} \widehat{\boldsymbol{i}}$, where $\widehat{\boldsymbol{i}}$ is a unit vector along the $i$ direction. In addition, $\sigma_{0}$ is a $2 \times 2$ identity matrix, and $\boldsymbol{\sigma}=\sum_{i} \sigma_{i} \widehat{\boldsymbol{i}}$ is a vector of Pauli spin matrices in such a way that $d_{\mathbf{k}}^{i}=\alpha_{i} k_{i}$ corresponds to the Weyl SOC when $\alpha_{i}=\alpha$ for all $i=\{x, y, z\}$, a Rashba SOC when $\alpha_{z}=0$, and to an ERD SOC when $\alpha_{y}=\alpha_{z}=0$. Here, we choose $\alpha \geqslant 0$ without the loss of generality. The resultant energy eigenvalues and eigenstates can be briefly denoted as $H_{\mathbf{k}}|s \mathbf{k}\rangle=\xi_{s \mathbf{k}}(\mathbf{h})|s \mathbf{k}\rangle$, where $\xi_{s \mathbf{k}}(\mathbf{h})=\xi_{\mathbf{k}}+s Z_{\mathbf{k}}(\mathbf{h})$ with $s= \pm$ and $Z_{\mathbf{k}}(\mathbf{h})=\left|\mathbf{Z}_{\mathbf{k}}(\mathbf{h})\right|$ is the helicity spectrum.

Expanding the corresponding thermodynamic potential $\Omega(\mathbf{h})=-T \sum_{s \mathbf{k}} \ln \left[1+e^{-\xi_{s \mathbf{k}}(\mathbf{h}) / T}\right]$ in powers of $\mathbf{h}$, where $T$ is the temperature (in units of $k_{B} \rightarrow 1$ the Boltzmann constant), i.e., $\Omega(\mathbf{h})=\Omega(\mathbf{0})-(1 / 2) \sum_{i j} \chi_{i j} h_{i} h_{j}+\cdots$, we identify the spin-susceptibility tensor as $\chi_{i j} \stackrel{\mathbf{h} \rightarrow \mathbf{0}}{=}-\partial^{2} \Omega /\left(\partial h_{i} \partial h_{j}\right)$. For noninteracting Fermi gases, a compact way to express this 
result is as follows:

$$
\chi_{i j}=\frac{1}{2} \sum_{s s^{\prime} \mathbf{k}} \frac{\mathcal{X}_{s \mathbf{k}}-\mathcal{X}_{s^{\prime} \mathbf{k}}}{\xi_{s \mathbf{k}}-\xi_{s^{\prime} \mathbf{k}}} \operatorname{Re}_{\mathbf{h} \rightarrow \mathbf{0}}\left\langle s\left|\frac{\partial H_{\mathbf{k}}}{\partial h_{i}}\right| s^{\prime}\right\rangle\left\langle s^{\prime}\left|\frac{\partial H_{\mathbf{k}}}{\partial h_{j}}\right| s\right\rangle,
$$

where $\mathcal{X}_{s \mathbf{k}}=\tanh \left[\xi_{s \mathbf{k}} /(2 T)\right]$ is a thermal factor with $\xi_{s \mathbf{k}}=$ $\xi_{\mathbf{k}}+s d_{\mathbf{k}}$ and $d_{\mathbf{k}}=\left|\mathbf{d}_{\mathbf{k}}\right|$, Re is the real part, and $|s\rangle \equiv|s \mathbf{k}\rangle$ is adapted for a simpler notation. Here, the derivative notion is implied for the $s \rightarrow s^{\prime}$ terms. To gain further insight, we separate $\chi_{i j}=\chi_{i j}^{\text {intra }}+\chi_{i j}^{\text {inter }}$ into its intraband and interband contributions as follows:

$$
\begin{gathered}
\chi_{i j}^{\text {intra }}=\frac{1}{4 T} \sum_{s \mathbf{k}} \frac{d_{\mathbf{k}}^{i} d_{\mathbf{k}}^{j}}{d_{\mathbf{k}}^{2}} \mathcal{Y}_{s \mathbf{k}}, \\
\chi_{i j}^{\text {inter }}=\frac{1}{2} \sum_{s \mathbf{k}} \frac{\mathcal{X}_{s \mathbf{k}}}{s d_{\mathbf{k}}}\left(\delta_{i j}-\frac{d_{\mathbf{k}}^{i} d_{\mathbf{k}}^{j}}{d_{\mathbf{k}}^{2}}\right),
\end{gathered}
$$

where $\mathcal{Y}_{s \mathbf{k}}=\operatorname{sech}^{2}\left[\xi_{s \mathbf{k}} /(2 T)\right]$ is another thermal factor, and $\delta_{i j}$ is a Kronecker delta. While the intraband term corresponds to the Pauli-paramagnetic contribution, the interband term is of Van Vleck-type accounting for the field-induced virtual transitions between the helicity bands [3-7]. Note that we trivially obtain the usual Pauli expression $\chi_{i j}=\delta_{i j} \sum_{\mathbf{k}} \mathcal{Y}_{\mathbf{k}} /(2 T)$ of a free Fermi gas when $d_{\mathbf{k}}=0$.

Analytical understanding of these intraband and interband contributions in the ground state proves to be particularly illuminating for the analysis of our numerical results that are presented in Sec. IV for the SF Fermi gases. Thus, by setting $T \rightarrow 0$, we obtain $\chi_{i j}^{\text {intra }}=\sum_{s \mathbf{k}} d_{\mathbf{k}}^{i} d_{\mathbf{k}}^{j} \delta\left(\xi_{s \mathbf{k}}\right) / d_{\mathbf{k}}^{2}$ and $\chi_{i j}^{\text {inter }}=\sum_{s \mathbf{k}} s \theta\left(\xi_{s \mathbf{k}}\right) /\left(\delta_{i j} / d_{\mathbf{k}}-d_{\mathbf{k}}^{i} d_{\mathbf{k}}^{j} / d_{\mathbf{k}}^{3}\right)$, where $\delta(x)$ is the Dirac delta function and $\theta(x)$ is the Heaviside step function. For instance, these expressions lead to $\chi_{i j}^{\text {intra }}=\delta_{i j} \theta(\mu+$ $\left.m \alpha^{2} / 2\right)\left(N / \epsilon_{F}\right)\left[\mu /\left(2 \epsilon_{F}\right)+m^{2} \alpha^{2} / k_{F}^{2}\right] / \sqrt{\mu / \epsilon_{F}+m^{2} \alpha^{2} / k_{F}^{2}}$ and $\chi_{i j}^{\text {inter }}=\delta_{i j} \theta\left(\mu+m \alpha^{2} / 2\right)\left(N / \epsilon_{F}\right) \sqrt{\mu / \epsilon_{F}+m^{2} \alpha^{2} / k_{F}^{2}}$ for the Weyl SOC in three dimensions, and $\chi_{z z}^{\text {intra }}=0,2 \chi_{\perp}^{\text {intra }}=$ $\theta(\mu) N / \epsilon_{F}+\theta(-\mu) \theta\left(\mu+m \alpha^{2} / 2\right)\left(N / \epsilon_{F}\right) / \sqrt{1+2 \mu /\left(m \alpha^{2}\right)}$ and $\quad \chi_{z z}^{\text {inter }}=2 \chi_{\perp}^{\text {inter }}=\theta(\mu) N / \epsilon_{F}+\theta(-\mu) \theta(\mu+$ $\left.m \alpha^{2} / 2\right)\left(N / \epsilon_{F}\right) \sqrt{1+2 \mu /\left(m \alpha^{2}\right)}$ for the Rashba SOC in two dimensions. Here, $N=k_{F}^{3} V /\left(3 \pi^{2}\right)$ and $k_{F}^{2} A /(2 \pi)$ are the total number of particles in three and two dimensions, respectively, with $\epsilon_{F}=k_{F}^{2} /(2 m)$ the corresponding Fermi energy. Thus, in the $\alpha \rightarrow 0^{+}$limit, we find $\chi_{i j}^{\text {intra }}=\delta_{i j} N /\left(2 \epsilon_{F}\right)$ and $\chi_{i j}^{\text {inter }}=\delta_{i j} N / \epsilon_{F}$ for the Weyl SOC in three dimensions, and $\chi_{z z}^{\text {intra }}=0,2 \chi_{\perp}^{\text {intra }}=N / \epsilon_{F}$ and $\chi_{z z}^{\text {inter }}=2 \chi_{\perp}^{\text {inter }}=N / \epsilon_{F}$ for the Rashba SOC in two dimensions. Note that these results are in agreement with the usual expression $\chi_{i j}=\delta_{i j} D N /\left(2 \epsilon_{F}\right)$ of a free Fermi gas, i.e., when $\mathbf{d}_{\mathbf{k}}=\mathbf{0}$, in $D$ dimensions.

Having shown that the interband contribution plays an equally important role in recovering the usual Pauli paramagnetism of a free Fermi gas in the $\mathbf{d}_{\mathbf{k}} \rightarrow \mathbf{0}$ limit, next we investigate its effects on the SF Fermi gases.

\section{SUPERFLUID FERMI GASES}

Assuming a zero-ranged density-density attraction between $\uparrow$ and $\downarrow$ particles in real space, we restrict ourselves to the uniform BCS description in $\mathbf{k}$ space that is governed by the mean-field Hamiltonian $H_{\mathrm{mf}}=(1 / 2) \sum_{\mathbf{k}} \Psi_{\mathbf{k}}^{\dagger} M_{\mathbf{k}} \Psi_{\mathbf{k}}+$ $\sum_{\mathbf{k}} \xi_{\mathbf{k}}+\Delta^{2} / U$. Here, the spinor operator $\Psi_{\mathbf{k}}^{\dagger}=\left(\psi_{\mathbf{k}}^{\dagger} \psi_{-\mathbf{k}}\right)$ with $\psi_{\mathbf{k}}^{\dagger}=\left(\psi_{\uparrow_{\mathbf{k}}}^{\dagger} \psi_{\downarrow \mathbf{k}}^{\dagger}\right)$ creates (annihilates) particles (holes) that are characterized by the following matrix:

$$
M_{\mathbf{k}}=\left[\begin{array}{cc}
\xi_{\mathbf{k}} \sigma_{0}-\mathbf{Z}_{\mathbf{k}}(\mathbf{h}) \cdot \boldsymbol{\sigma} & i \Delta \sigma_{y} \\
-i \Delta \sigma_{y} & -\xi_{\mathbf{k}} \sigma_{0}+\mathbf{Z}_{-\mathbf{k}}(\mathbf{h}) \cdot \boldsymbol{\sigma}^{*}
\end{array}\right],
$$

where the BCS mean field $\Delta=U\left\langle\psi_{\uparrow \mathbf{k}} \psi_{\downarrow-\mathbf{k}}\right\rangle$, with $U \geqslant 0$ the strength of the contact interaction and $\langle\cdots\rangle$ the thermal average, is taken as a real parameter without the loss of generality. Similar to the noninteracting Fermi gases discussed in Sec. II, one can in principle calculate $\chi_{i j}$ by taking the derivatives of the mean-field thermodynamic potential $\Omega(\mathbf{h})=$ $-(T / 2) \sum_{\lambda \mathbf{k}} \ln \left[1+e^{-E_{\lambda \mathbf{k}}(\mathbf{h}) / T}\right]+\sum_{\mathbf{k}} \xi_{\mathbf{k}}+\Delta^{2} / U$, where $\lambda=$ $\{1,2,3,4\}$ labels the quasiparticle and quasihole energies. While it is possible to simplify this expression in some special cases, i.e., by imposing the particle-hole symmetry $E_{\lambda \mathbf{k}}(\mathbf{h})=-E_{\lambda^{\prime},-\mathbf{k}}(\mathbf{h})$ of the system and the traceless condition $\sum_{\lambda} E_{\lambda \mathbf{k}}(\mathbf{h})=0$ for every $\mathbf{k}$, it is unfortunate that the spectra $E_{\lambda \mathbf{k}}(\mathbf{h})$ cannot be put into a closed-analytical (yet simple) form in the simultaneous presence of arbitrary $\mathbf{h}$ and $\mathbf{d}_{\mathbf{k}}$ fields.

To circumvent this problem, next we make use of the Green's function approach, which allows us to expand $\Omega(\mathbf{h})$ in powers of $\mathbf{h}$ in a systematic way [8]. For this purpose, we first note that $\Omega(\mathbf{0})=-(T / 2) \sum_{\ell \mathbf{k}} \ln \operatorname{det} G_{\ell \mathbf{k}}^{-1}+\sum_{\mathbf{k}} \xi_{\mathbf{k}}+\Delta^{2} / U$, where

$$
G_{\ell \mathbf{k}}^{-1}=\left[\begin{array}{cc}
\left(i \omega_{\ell}-\xi_{\mathbf{k}}\right) \sigma_{0}-\mathbf{d}_{\mathbf{k}} \cdot \boldsymbol{\sigma} & i \Delta \sigma_{y} \\
-i \Delta \sigma_{y} & \left(i \omega_{\ell}+\xi_{\mathbf{k}}\right) \sigma_{0}-\mathbf{d}_{\mathbf{k}} \cdot \boldsymbol{\sigma}^{*}
\end{array}\right]
$$

is the inverse Green's function for the $\mathbf{h}=\mathbf{0}$ problem $[1,2,4-8]$. Here, $\omega_{\ell}=2 \pi(\ell+1 / 2) T$ is the fermionic Matsubara frequency, and the quasiparticle and quasihole energies are determined by setting $\operatorname{det} G_{\ell \mathbf{k}}^{-1}=\left[\left(i \omega_{\ell}\right)^{2}-\right.$ $\left.E_{+, \mathbf{k}}^{2}\right]\left[\left(i \omega_{\ell}\right)^{2}-E_{-, \mathbf{k}}^{2}\right]$ to zero, where $E_{s \mathbf{k}}=\sqrt{\xi_{s \mathbf{k}}^{2}+\Delta^{2}}$ with $s= \pm$ are the quasiparticle energies. Similarly, we can write $\Omega(\mathbf{h})=-(T / 2) \sum_{\ell \mathbf{k}} \ln \operatorname{det} \mathcal{G}_{\ell \mathbf{k}}^{-1}+\sum_{\mathbf{k}} \xi_{\mathbf{k}}+\Delta^{2} / U$, where $\mathcal{G}_{\ell \mathbf{k}}^{-1}=G_{\ell \mathbf{k}}^{-1}-\Sigma_{\mathbf{h}}$ is the inverse Green's function for the $\mathbf{h} \neq \mathbf{0}$ problem, where the particle and hole components of $\Sigma_{\mathbf{h}}$ are $\Sigma_{\mathbf{h}}^{11}=-\mathbf{h} \cdot \boldsymbol{\sigma}, \Sigma_{\mathbf{h}}^{22}=\mathbf{h} \cdot \boldsymbol{\sigma}^{*}$, and $\Sigma_{\mathbf{h}}^{12}=\Sigma_{\mathbf{h}}^{21}=0$. Thus, we can formally expand the thermodynamic potential as $\Omega(\mathbf{h})=\Omega(\mathbf{0})+(T / 2) \sum_{n \ell \mathbf{k}} \operatorname{Tr}\left(G_{\ell \mathbf{k}} \Sigma_{\mathbf{h}}\right)^{n} / n$, where $\operatorname{Tr}$ denotes a trace over the particle/hole and spin sectors, and $n \geqslant 1$ is a positive integer. Here, the particle/hole components of the Green's function $G_{\ell \mathbf{k}}$ can be written as follows:

$$
\begin{aligned}
G_{\ell \mathbf{k}}^{11} & =\frac{1}{2} \sum_{s} \frac{i \omega_{\ell}+\xi_{s \mathbf{k}}}{\left(i \omega_{\ell}\right)^{2}-E_{s \mathbf{k}}^{2}}\left(\sigma_{0}+\frac{\mathbf{d}_{\mathbf{k}} \cdot \boldsymbol{\sigma}}{s d_{\mathbf{k}}}\right), \\
G_{\ell \mathbf{k}}^{22} & =\frac{1}{2} \sum_{s} \frac{i \omega_{\ell}-\xi_{s \mathbf{k}}}{\left(i \omega_{\ell}\right)^{2}-E_{s \mathbf{k}}^{2}}\left(\sigma_{0}-\frac{\mathbf{d}_{\mathbf{k}} \cdot \boldsymbol{\sigma}^{*}}{s d_{\mathbf{k}}}\right), \\
G_{\ell \mathbf{k}}^{12} & =\frac{1}{2} \sum_{s} \frac{-i \Delta \sigma_{y}}{\left(i \omega_{\ell}\right)^{2}-E_{s \mathbf{k}}^{2}}\left(\sigma_{0}-\frac{\mathbf{d}_{\mathbf{k}} \cdot \sigma^{*}}{s d_{\mathbf{k}}}\right), \\
G_{\ell \mathbf{k}}^{21} & =\frac{1}{2} \sum_{s} \frac{i \Delta \sigma_{y}}{\left(i \omega_{\ell}\right)^{2}-E_{s \mathbf{k}}^{2}}\left(\sigma_{0}+\frac{\mathbf{d}_{\mathbf{k}} \cdot \sigma}{s d_{\mathbf{k}}}\right),
\end{aligned}
$$

and they are obtained through a lengthy but a straightforward algebra, coinciding with the available literature [1,2,4-8]. Given the power-series expansion of $\Omega(\mathbf{h})$ with $\mathbf{h}$, we imme- 
diately identify $\chi_{i j} \stackrel{\mathbf{h} \rightarrow \mathbf{0}}{=}-\left[T /\left(2 h_{i} h_{j}\right)\right] \sum_{\ell \mathbf{k}} \operatorname{Tr}\left(G_{\ell \mathbf{k}} \Sigma_{\mathbf{h}}\right)^{2}$ as the spin-susceptibility tensor for the SF Fermi gases.

Using this prescription, and after some tedious algebra, we eventually obtain a rather simple expression for $\chi_{i j}=-T \sum_{s s^{\prime} \ell \mathbf{k}}\left\{\left[\left(i \omega_{\ell}\right)^{2}+\xi_{s \mathbf{k}} \xi_{s^{\prime} \mathbf{k}}+\Delta^{2}\right] /\right.$ $\left.\operatorname{det} G_{\ell \mathbf{k}}^{-1}\right\} \operatorname{Re}_{\mathbf{h} \rightarrow \mathbf{0}}\left\langle s\left|\partial H_{\mathbf{k}} / \partial h_{i}\right| s^{\prime}\right\rangle\left\langle s^{\prime}\left|\partial H_{\mathbf{k}} / \partial h_{j}\right| s\right\rangle$, where $H_{\mathbf{k}}$ is the noninteracting Hamiltonian density introduced in Sec. II. Furthermore, performing the summation over the Matsubara frequency $\omega_{\ell}$, a compact way to express the final result is as follows:

$$
\begin{aligned}
\chi_{i j}= & \frac{1}{2} \sum_{s s^{\prime} \mathbf{k}} \frac{\frac{\mathcal{X}_{\mathbf{s}}}{E_{s \mathbf{k}}}\left(\xi_{s \mathbf{k}}+\frac{2 \Delta^{2}}{\xi_{s \mathbf{k}}+\xi_{s^{\prime} \mathbf{k}}}\right)-\frac{\mathcal{X}_{s^{\prime} \mathbf{k}}}{E_{s^{\prime} \mathbf{k}}}\left(\xi_{s^{\prime} \mathbf{k}}+\frac{2 \Delta^{2}}{\xi_{s^{\prime} \mathbf{k}}+\xi_{s \mathbf{k}}}\right)}{\xi_{s \mathbf{k}}-\xi_{s^{\prime} \mathbf{k}}} \\
& \times \operatorname{Re}_{\mathbf{h} \rightarrow \mathbf{0}}\left\langle s\left|\frac{\partial H_{\mathbf{k}}}{\partial h_{i}}\right| s^{\prime}\right\rangle\left\langle s^{\prime}\left|\frac{\partial H_{\mathbf{k}}}{\partial h_{j}}\right| s\right\rangle,
\end{aligned}
$$

where $\mathcal{X}_{s \mathbf{k}}=\tanh \left[E_{s \mathbf{k}} /(2 T)\right]$ is a thermal factor. Here, the derivative notion is again implied for the $s \rightarrow s^{\prime}$ terms following the analogous treatment of the noninteracting case. In addition, we again separate $\chi_{i j}=\chi_{i j}^{\mathrm{intra}}+\chi_{i j(a)}^{\mathrm{inter}}+\chi_{i j(b)}^{\mathrm{inter}}$ into its intraband and interband contributions as follows:

$$
\begin{gathered}
\chi_{i j}^{\text {intra }}=\frac{1}{4 T} \sum_{s \mathbf{k}} \frac{d_{\mathbf{k}}^{i} d_{\mathbf{k}}^{j}}{d_{\mathbf{k}}^{2}} \mathcal{Y}_{s \mathbf{k}}, \\
\chi_{i j(a)}^{\text {inter }}=\frac{1}{2} \sum_{s \mathbf{k}} \frac{\xi_{s \mathbf{k}} \mathcal{X}_{s \mathbf{k}}}{s d_{\mathbf{k}} E_{s \mathbf{k}}}\left(\delta_{i j}-\frac{d_{\mathbf{k}}^{i} d_{\mathbf{k}}^{j}}{d_{\mathbf{k}}^{2}}\right), \\
\chi_{i j(b)}^{\text {inter }}=\frac{\Delta^{2}}{2} \sum_{s \mathbf{k}} \frac{\mathcal{X}_{s \mathbf{k}}}{s d_{\mathbf{k}} \xi_{\mathbf{k}} E_{s \mathbf{k}}}\left(\delta_{i j}-\frac{d_{\mathbf{k}}^{i} d_{\mathbf{k}}^{j}}{d_{\mathbf{k}}^{2}}\right),
\end{gathered}
$$

where $\mathcal{Y}_{s \mathbf{k}}=\operatorname{sech}^{2}\left[E_{s \mathbf{k}} /(2 T)\right]$ is another thermal factor. Note that we trivially obtain the usual BCS expression $\chi_{i j}=$ $\delta_{i j} \sum_{\mathbf{k}} \mathcal{Y}_{\mathbf{k}} /(2 T)$ of a SF Fermi gas when $d_{\mathbf{k}}=0$. These closedform analytical tensors, that are generalized for an arbitrary SOC field $\mathbf{d}_{\mathbf{k}}$, are the main results of this paper. It can be readily verified that their total response tensor $\chi_{i j}$ reproduces all known limits in the cold-atom literature, e.g., the isotropic Weyl SOC [8] and the $z z$ component of the Rashba SOC [10] at $T=0$. As discussed in Sec. IV, while $\chi_{i j(a)}^{\mathrm{inter}}$ is a paramagnetic contribution, $\chi_{i j(b)}^{\text {inter }}$ is a diamagnetic one in such a way that their sums eventually cancel each other precisely toward the molecular gas limit. This competition reveals the physical mechanism that forms spinless molecules from Cooper pairs in the BEC limit, which naturally exhibit zero spin susceptibility at any $T$ including their ground state.

More importantly, while Eqs. (9) and (10) evolve, respectively, from Eqs. (1) and (2) of the noninteracting problem as soon as $\Delta \neq 0$, it is only Eq. (11) that uniquely contributes to the spin-susceptibility tensor of SF Fermi gases. It is intriguing to note that Eq. (11) can be put into precisely the same form as the interband contribution to the SF density tensor $\rho_{i j}^{\text {inter }}=-\left(m \Delta^{2} / V\right) \sum_{s \mathbf{k}} d_{\mathbf{k}} \mathcal{X}_{s \mathbf{k}} g_{\mathbf{k}}^{i j} /\left(s \xi_{\mathbf{k}} E_{s \mathbf{k}}\right)$ [16] and the pair mass tensor $c_{i j}^{\text {inter }}=-(1 / 4) \sum_{s \mathbf{k}} d_{\mathbf{k}} \mathcal{X}_{s \mathbf{k}} g_{\mathbf{k}}^{i j} /\left(s \xi_{\mathbf{k}} \xi_{s \mathbf{k}}\right)$ near $T \rightarrow T_{c}$ [17]. For instance, if the SOC field is of the form $d_{\mathbf{k}}^{i}=\alpha_{i} k_{i}$, then we can reexpress Eq. (11) as $\chi_{i j(b)}^{\text {inter }}=$ $\left[\Delta^{2} /\left(\alpha_{i} \alpha_{j}\right)\right] \sum_{s \mathbf{k}} d_{\mathbf{k}} \mathcal{X}_{s \mathbf{k}} g_{\mathbf{k}}^{i j} /\left(s \xi_{\mathbf{k}} E_{s \mathbf{k}}\right)$, where $g_{\mathbf{k}}^{i j}$ is the total quantum metric of the helicity bands. What allows this cu- rious correspondence is that the total quantum metric $g_{\mathbf{k}}^{i j}=$ $\left[\sum_{\ell}\left(\partial d_{\mathbf{k}}^{\ell} / \partial k_{i}\right)\left(\partial d_{\mathbf{k}}^{\ell} / \partial k_{j}\right)-\left(\partial d_{\mathbf{k}} / \partial k_{i}\right)\left(\partial d_{\mathbf{k}} / \partial k_{j}\right)\right] /\left(2 d_{\mathbf{k}}^{2}\right)$ of the helicity bands reduces to $g_{\mathbf{k}}^{i j}=\alpha_{i} \alpha_{j}\left(d_{\mathbf{k}}^{2} \delta_{i j}-d_{\mathbf{k}}^{i} d_{\mathbf{k}}^{j}\right) /\left(2 d_{\mathbf{k}}^{4}\right)$ for the specific case when $d_{\mathbf{k}}^{i}=\alpha_{i} k_{i}[16,17]$. Thus, we identify $\rho_{i j}^{\text {inter }}=-(m / V) \alpha_{i} \alpha_{j} \chi_{i j(b)}^{\text {inter }}$ for such SOC fields at any $T$. From the quantum geometric point of view, this identification reveals that the interband term Eq. (11) may be interpreted as the quantum metric contribution to the spin-susceptibility tensor, caused by the geometric effects on the Cooper pairs. Likewise, the remaining interband term Eq. (10) may also be interpreted as the quantum metric contribution to the spin-susceptibility tensor, caused by the geometric effects on the single particles.

The existence of a nonzero ground-state spin response at $T=0$ is not truly a direct measure of the SOC-induced spin-triplet component of the SF order parameter. Our analysis reveals that, unlike the interband term Eq. (11) for the Cooper pairs, the Van Vleck-type interband term Eq. (10) has a nonzero contribution even in the normal state regardless of whether $\Delta=0$. Indeed, next we show that the paramagnetic contribution coming from Eq. (10) dominates the diamagnetic one coming from Eq. (11) for most of the parameter regimes of experimental interest.

\section{NUMERICAL RESULTS}

It is clear that the numerical evaluations of Eqs. (9), (10), and (11) necessitate a priori the solutions of $\Delta$ and $\mu$ for any given set of $U, \mathbf{d}_{\mathbf{k}}$, and $T$ parameters. Within the BCS mean-field approach, this is achieved through the iterative solutions of the self-consistency equations for the SF order parameter $1 / U=\sum_{s \mathbf{k}} \mathcal{X}_{s \mathbf{k}} /\left(4 V E_{s \mathbf{k}}\right)$, and the total number of particles $N=\sum_{s \mathbf{k}}\left[1 / 2-\xi_{s \mathbf{k}} \mathcal{X}_{s \mathbf{k}} /\left(2 E_{s \mathbf{k}}\right)\right]$. In accordance with the cold-atom literature, while we substitute the theoretical parameter $U$ with the experimentally more relevant two-body scattering length $a_{s}$ in vacuum via the usual relation $V / U=$ $-m V /\left(4 \pi a_{s}\right)+\sum_{\mathbf{k}} 1 /\left(2 \epsilon_{\mathbf{k}}\right)$ for three-dimensional systems, we substitute $U$ with the two-body binding energy $\epsilon_{b} \geqslant 0$ in vacuum via the usual relation $A / U=\sum_{\mathbf{k}} 1 /\left(2 \epsilon_{\mathbf{k}}+\epsilon_{b}\right)$ for two-dimensional ones.

Since the mean-field approach works best at low $T$ in the entire BCS-BEC crossover regime, next we present our numerical calculations only for the SF ground state as it sufficiently illustrates our main findings in this paper. For this purpose, we may set $\mathcal{X}_{s \mathbf{k}} \rightarrow 1$ and $\mathcal{Y}_{s \mathbf{k}} \rightarrow 0$ for every $s$ and $\mathbf{k}$ as long as $\Delta \neq 0$ in the $T \rightarrow 0$ limit, leading to a vanishing intraband contribution $\chi_{i j}^{\text {intra }}=0$ for any $\mathbf{d}_{\mathbf{k}}$. Thus, in the SF ground state, while the interband spin-susceptibility tensor is isotropic, $\chi_{i j}^{\text {inter }}=\chi \delta_{i j}$, for the Weyl SOC [8], its diagonal components are related by $\chi_{z z}^{\text {inter }}=2 \chi_{\perp}^{\text {inter }}$ for the Rashba SOC [9], and by $\chi_{x x}^{\text {inter }}=0$ and $\chi_{z z}^{\text {inter }}=\chi_{y y}^{\text {inter }}$ for the ERD SOC [9] in three dimensions. However, in two dimensions, the diagonal components are related by $\chi_{z z}^{\text {inter }}=2 \chi_{\perp}^{\text {inter }}$ for the Rashba SOC, and by $\chi_{x x}^{\text {inter }}=0$ and $\chi_{z z}^{\text {inter }}=\chi_{y y}^{\text {inter }}$ for the ERD SOC. Thus, as all of the nontrivial components are proportional to each other for all SOC fields considered in this paper, next we present only the $z z$ component at $T=0$.

In Fig. 1, we show colored maps of $\chi_{z z} \equiv \chi_{z z}^{\text {inter }}=\chi_{z z(a)}^{\text {inter }}+$ $\chi_{z z(b)}^{\text {inter }}$ for the Weyl, Rashba, and ERD SOCs introduced in 


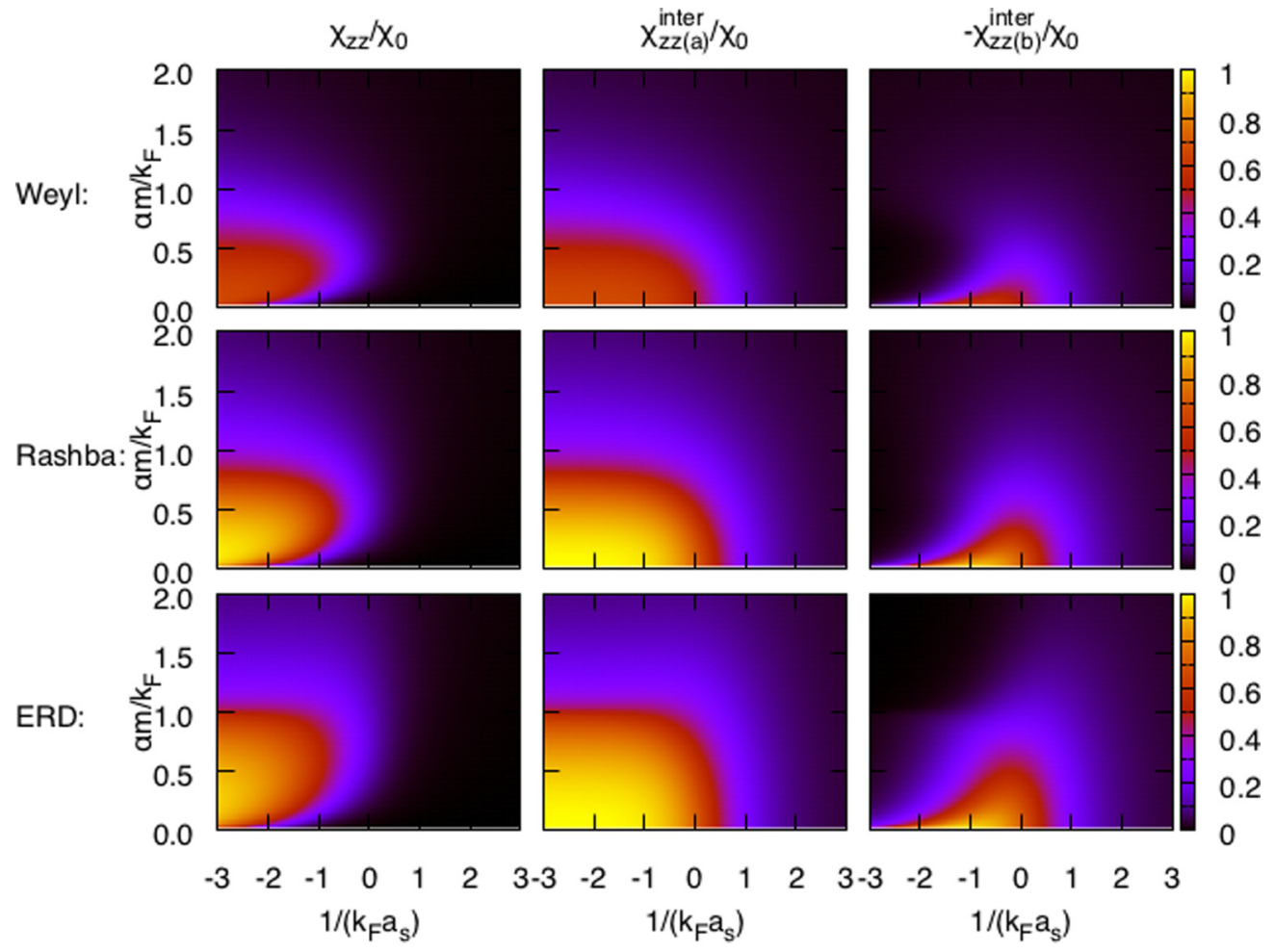

FIG. 1. The $z z$ component of the spin-susceptibility tensor is mapped [in units of the free Fermi gas value $\chi_{0}=3 N /\left(2 \epsilon_{F}\right)$ ] for a threedimensional SF Fermi gas in the ground state. Since $\chi_{z z}^{\text {intra }}=0$ as long as $\Delta \neq 0$ at $T=0$, we show $\chi_{z z}=\chi_{z z(a)}^{\text {inter }}+\chi_{z z(b)}^{\text {inter }}$ together with the competing interband contributions coming from the paramagnetic term $\chi_{z z(a)}^{\text {inter }}$ and the diamagnetic term $\chi_{z z(b)}^{\text {inter }}$. Note that this competition gives rise to a cancellation of the spin response $\chi_{z z} \rightarrow 0$ for the molecular SF Bose gas.

Sec. II, along with the specific contributions from $\chi_{z z(a)}^{\text {inter }}$ and $-\chi_{z z(b)}^{\text {inter }}$. See Fig. 2 for the analogous results in two dimensions. The strengths $\alpha$ of the SOC fields are varied in both figures for the entire BCS-BEC crossover. First, we verify that the peak values of $\chi_{z z}$ occurring in the BCS limit are consistent with the $\chi_{z z}^{\text {inter }}$ contributions that are discussed in Sec. II for

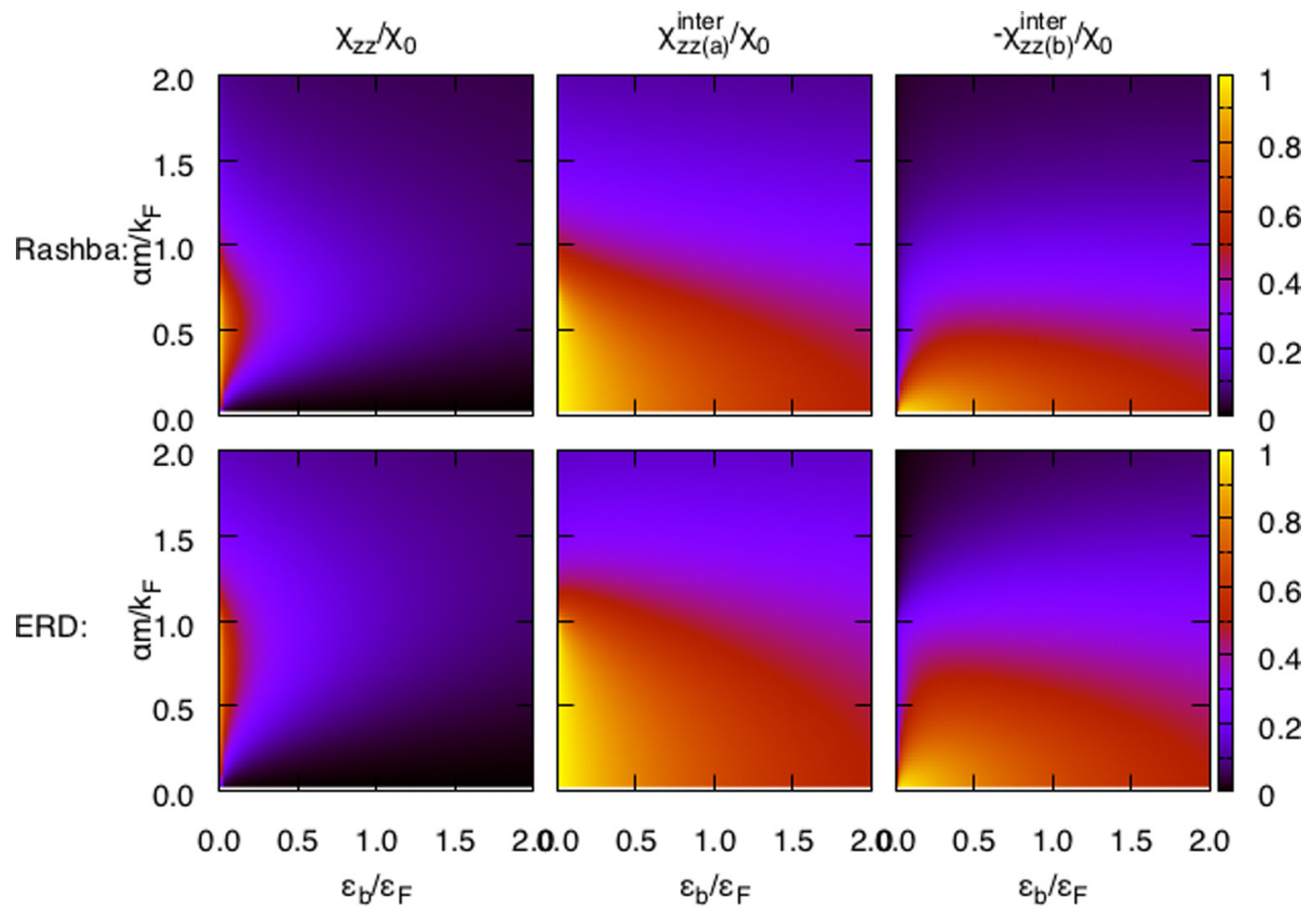

FIG. 2. Same as Fig. 1 but for a two-dimensional SF Fermi gas, where $\chi_{0}=N / \epsilon_{F}$. 
the noninteracting Fermi gases. For instance, $\chi_{z z}^{\text {inter }}=N / \epsilon_{F}$ for the Weyl SOC in three dimensions as well as for the Rashba SOC in two dimensions when $\alpha \rightarrow 0^{+}$. Second, we observe that $\chi_{z z}$ is dominated by the paramagnetic contribution $\chi_{z z(a)}^{\text {inter }}$, and never changes sign for the entire parameter space. In fact, the magnitude of the diamagnetic contribution $\left|\chi_{z z(b)}^{\text {inter }}\right|$ turns out to be bounded by $\chi_{z z(a)}^{\mathrm{inter}}$ in the spinless limit of a molecular SF Bose gas in such a way that their competition gives rise to the complete cancellation of the spin response where $\chi_{z z} \rightarrow 0$.

Furthermore, our numerical results clarify the origins of the nonzero spin response that is caused by SOC in a variety of noncentrosymmetric superconductors and SFs in their ground state. Since $\chi_{z z(b)}^{\text {inter }}$ is not the leading contribution to $\chi_{z z}$ for most of the parameter space, $\chi_{z z} \neq 0$ is clearly not primarily caused by the SOC induced spin-triplet component of the SF order parameter. In the condensed-matter literature [2-7], Eq. (11) is typically neglected and Eq. (10) is replaced with its normal-state value. This treatment works reasonably well for weak-coupling BCS superconductors and SFs, for which Eq. (11) has negligible contribution and Eq. (10) approximates the noninteracting result. These are clearly illustrated by our extensive numerical results. Away from the BCS regime, however, one needs to treat both contributions on an equal footing in order to recover the proper molecular limit, which is best seen in Fig. 2.

\section{CONCLUSIONS}

In summary, here we analyzed the spin response of spinorbit coupled Fermi SFs with arbitrary SOC and Zeeman fields. For this purpose, assuming a self-consistent mean-field approach for the BCS-BEC crossover problem, we derive a closed-form analytical expression for the generalized spinsusceptibility tensor $\chi_{i j}$ through the derivative expansion of the thermodynamic potential with the Green's function approach.
The tensor has three distinct contributions denoted as $\chi_{i j}=$ $\chi_{i j}^{\text {intra }}+\chi_{i j(a)}^{\text {inter }}+\chi_{i j(b)}^{\text {inter }}$. In addition to the usual paramagnetic Pauli intrahelicity contribution $\chi_{i j}^{\text {intra }}$ and the paramagnetic Van Vleck-type interhelicity contribution $\chi_{i j(a)}^{\text {inter }}$ that originate from their normal-state counterparts upon pairing, we found a diamagnetic interhelicity contribution $\chi_{i j(b)}^{\text {inter }}$ that is unique to the SF state. Motivated by the recent identification of some interband effects as the quantum metric contributions to the SF density and the Cooper pair mass [16,17], we noted that $\chi_{i j(a)}^{\text {inter }}$ and $\chi_{i j(b)}^{\text {inter }}$ contributions may be interpreted as geometric effects on the single particles and Cooper pairs, respectively. However, such a geometric interpretation holds only for those SOC fields that are of the generic form $\mathbf{d}_{\mathbf{k}}=\sum_{i} \alpha_{i} k_{i} \widehat{\boldsymbol{i}}$.

Furthermore, our extensive numerical calculations for the Weyl, Rashba, and ERD SOCs illustrated that it is the diamagnetic contribution $\chi_{i j(b)}^{\text {inter }}$ that grows gradually with pairing and cancels precisely the paramagnetic contribution $\chi_{i j(a)}^{\mathrm{inter}}$ away from the BCS regime. Thus, this competition revealed the physical mechanism that forms spinless molecules from Cooper pairs in the BEC limit, whose spin response naturally must vanish. The existence of a nonzero ground-state spin response is not truly a direct measure of the SOC-induced spin-triplet component of the SF order parameter. Our thorough analysis revealed that, unlike the $\chi_{i j(b)}^{\text {inter }}$ contribution that is unique to the SF state, the $\chi_{i j(a)}^{\mathrm{inter}}$ contribution is nonzero even in the normal ground state. Moreover, we showed that the spin response is dominated quite strongly by its normal-state counterpart $\chi_{i j(a)}^{\text {inter }}$ for most of the parameter regimes of interest. Note that since the $\chi_{i j(b)}^{\text {inter }}$ contribution has a strong peak in the vicinity of unitarity, observation of its diamagnetic effect is within the reach of ongoing cold-atom experiments [11-15].

\section{ACKNOWLEDGMENTS}

The author acknowledges support from TÜBITAK and the BAGEP award of the Turkish Science Academy.
[1] V. M. Edelstein, Magnetoelectric Effect in Polar Superconductors, Phys. Rev. Lett. 75, 2004 (1995).

[2] L. P. Gorkov and E. I. Rashba, Superconducting 2D System with Lifted Spin Degeneracy: Mixed Singlet-Triplet State, Phys. Rev. Lett. 87, 037004 (2001).

[3] S. K. Yip, Two-dimensional superconductivity with strong spinorbit interaction, Phys. Rev. B 65, 144508 (2002).

[4] P. A. Frigeri, D. F. Agterberg, and M. Sigrist, Spin susceptibility in superconductors without inversion symmetry, New J. Phys. 6, 115 (2004).

[5] K. V. Samokhin, Spin susceptibility of noncentrosymmetric superconductors, Phys. Rev. B 76, 094516 (2007).

[6] V. P. Mineev, Recent developments in unconventional superconductivity theory, J. Low. Temp. Phys. 158, 615 (2010).

[7] See the recent review by M. Smidman, M. B. Salamon, H. Q. Yuan, and D. F. Agterberg, Superconductivity and spinorbit coupling in non-centrosymmetric materials: A review, Rep. Prog. Phys. 80, 036501 (2017).

[8] L. He and X.-G. Huang, BCS-BEC crossover in threedimensional Fermi gases with spherical spin-orbit coupling, Phys. Rev. B 86, 014511 (2012).
[9] L. Han and C. A. R. Sá de Melo, Evolution from BCS to BEC superfluidity in the presence of spin-orbit coupling, Phys. Rev. A 85, 011606(R) (2012).

[10] H.-K. Tang, X. Yang, J. Sun, and H.-Q. Lin, BerezinskiiKosterlitz-Thouless phase transition of spin-orbit coupled Fermi gas in optical lattice, Europhys. Lett. 107, 40003 (2014).

[11] P. Wang, Z.-Q. Yu, Z. Fu, J. Miao, L. Huang, S. Chai, H. Zhai, and J. Zhang, Spin-Orbit Coupled Degenerate Fermi Gases, Phys. Rev. Lett. 109, 095301 (2012).

[12] L. W. Cheuk, A. T. Sommer, Z. Hadzibabic, T. Yefsah, W. S. Bakr, and M. W. Zwierlein, Spin-Injection Spectroscopy of a Spin-Orbit Coupled Fermi Gas, Phys. Rev. Lett. 109, 095302 (2012).

[13] R. A. Williams, M. C. Beeler, L. J. LeBlanc, K. Jiménez-García, and I. B. Spielman, Raman-Induced Interactions in a SingleComponent Fermi Gas Near an S-Wave Feshbach Resonance, Phys. Rev. Lett. 111, 095301 (2013).

[14] L. Huang, Z. Meng, P. Wang, P. Peng, S.-L. Zhang, L. Chen, D. Li, Q. Zhou, and J. Zhang, Experimental realization of a two-dimensional synthetic spin-orbit coupling in ultracold Fermi gases, Nat. Phys. 12, 540 (2016). 
[15] Z. Meng, L. Huang, P. Peng, D. Li, L. Chen, Y. Xu, C. Zhang, P. Wang, and J. Zhang, Experimental Observation of a Topological Band Gap Opening in Ultracold Fermi Gases with Two-Dimensional Spin-Orbit Coupling, Phys. Rev. Lett. 117, 235304 (2016).
[16] M. Iskin, Exposing the quantum geometry of spin-orbit coupled Fermi superfluids, arXiv:1711.07262.

[17] M. Iskin, Quantum metric contribution to the pair mass in spin-orbit coupled Fermi superfluids, Phys. Rev. A 97, 033625 (2018). 